\title{
9.02
}

\section{El consentimiento informado en sentencia del Tribunal Constitucional Español 37/2011 de 28 de marzo. ${ }^{1}$}

Informed consent at the sentence of the Spanish Constitutional Court 37/2011, march 28.

\author{
Angel Pelayo González-Torre \\ Profesor Titular de la Universidad de Cantabria. Santander, España.
}

Resumen: El Tribunal Constitucional considera incluido dentro del derecho fundamental a la integridad física y moral del art. 15 una facultad de oposición a la asistencia médica, de manera que el derecho se viola cuando se impone a una persona asistencia médica contra su voluntad. Que el artículo 15 no aluda al consentimiento informado no implica que este instituto quede fuera de la previsión constitucional, de manera que la garantía de la efectividad del derecho a la integridad personal implica en el ámbito médico que cualquier actuación que afecte a la integridad personal ha de ser consentida por el sujeto o estar constitucionalmente justificada. En este campo el derecho a la información alcanza una relevancia que hace que su omisión o defectuosa realización pueda suponer una lesión del derecho fundamental. Este derecho no puede verse limitado de manera injustificada como consecuencia de una situación de enfermedad, ya que este derecho es distinto del derecho a la salud y del derecho a la vida.

Palabras clave: Consentimiento informado; derecho fundamental; relación médicopaciente; información médica.

Key-words: Informed Consent; fundamental right; patient-professional relationship; medical information.

\section{Planteamiento.}

El Tribunal Constitucional va a abordar el tema del consentimiento informado a los tratamientos médicos de manera expresa y detallada por vez primera en su STC (Sala segunda), 37/2011 de 28 de marzo. Teniendo como ponente a la Magistrada Doña Elisa Pérez Vera.

\footnotetext{
${ }^{1}$ Este artículo ha sido elaborado en el marco del Proyecto HURI-AGE, El tiempo de los derechos, del Ministerio de Educación del Gobierno de España.
} 
La transcendencia de dicha sentencia del Tribunal Constitucional radica en que va a dejar sentado que el consentimiento informado es una consecuencia implícita y obligada de la garantía del derecho fundamental a la integridad física y moral el artículo 15 de la Constitución Española.

En cuanto al supuesto de hecho que va a dar lugar a este pronunciamiento judicial nos encontramos con un paciente al que se le realizó un cateterismo cardiaco sin informarle previamente de los riesgos que entrañaba la operación, y de resultas de los cuales quedo con una pérdida funcional total de su mano derecha. Habiendo denunciado el hecho su demanda fue rechazada en las dos primeras instancias, por lo que acude ahora en amparo ante el Tribunal Constitucional, ante el cual denuncia la vulneración de su derecho a la tutela judicial efectiva del artículo 24.1 de la Constitución en relación con los derechos a la integridad física del artículo 15 y a la libertad protegida en el artículo 17.1 de la Constitución, por habérsele denegado el derecho a ser indemnizado por la pérdida funcional total de la mano derecha.

En su recurso el paciente considera que el resultado dañoso se debió o bien a una mala praxis en la intervención o bien a la absoluta falta de información previa a la misma sobre sus posibles riesgos o sobre las vías alternativas para la práctica del cateterismo, ya que por toda información lo único que obtuvo fue el documento con las instrucciones pertinentes para el alta ${ }^{2}$.

En este punto el reclamante pone de manifiesto el desconocimiento respecto del origen del daño causado. No sabe si deriva de una mala práctica, ya que ignora cómo de adecuadamente se realizo la intervención desde el punto de vista técnico. Como sabemos la relación de causalidad entre la actuación médica y el daño es un elemento especialmente difícil de concretar en el caso de las actuaciones sanitarias. Esto es así tanto por tratarse de una actuación técnica con frecuencia compleja, y cuya corrección ha de ser valorada por otros profesionales sanitarios, como por la presencia de lo que se denomina el alea, en referencia a lo indeterminado, lo imprevisible en cualquier actuación sanitaria derivado de la particular constitución del paciente o de su concreto estado físico y que puede determinar que reaccione al tratamiento o la intervención de un modo inesperado. El alea, como concepto acuñado jurisprudencialmente, es un elemento que se considera de posible

\footnotetext{
${ }^{2}$ Cfr. Antecedente 2b.
} 
concurrencia en muchas actuaciones sanitarias, y resulta muy útil para poner en cuestión la relación de causalidad entre la actuación sanitaria y el resultado dañoso, al sembrar la duda en cuanto a que el resultado se deba a factores imprevisibles derivados del particular estado físico del sujeto y no a la actuación del profesional.

Por eso el recurrente alega la falta de información como elemento alternativo capaz de conseguir la responsabilización, con independencia de la buena o mala actuación profesional. Entiende que la falta de consentimiento e información implica que no debe asumir los daños derivados de la mala praxis o de la materialización de riesgos. Con ello se pone de manifiesto el uso del consentimiento informado como arma procesal. La falta de consentimiento resulta sin duda más fácil de probar, y no requiere tener que acudir a peritajes profesionales como ocurre con la relación de causalidad actuación médica-resultado dañoso. Además la carga de su prueba recae sobre quienes llevaron a cabo la actuación sanitaria, con lo que se invierte a favor del actor respecto del sistema clásico de responsabilidad por daños, aumentando las posibilidades de conseguir un pronunciamiento incriminatorio.

En cuanto al itinerario procesal seguido por la pretensión del paciente, la sentencia del Juzgado de primera instancia había considerado probado que no se facilitó información al paciente, ni por parte de los médicos que llevaron a cabo la intervención ni por parte de los médicos de la UVI que le atendieron. No obstante esta instancia desestima la demanda considerando que en el caso de autos el hecho de que hubiera tenido años antes una intervención del mismo tipo, unido a la urgencia relativa de la intervención, permiten considerar que realmente no se privó al demandante de una información esclarecedora previa al consentimiento que dé lugar a la procedencia de ser indemnizado ${ }^{3}$.

En igual sentido se pronunciará la Audiencia Provincial de Bizkaia desestimando el recurso contra la sentencia de primera instancia en la que se insiste sobre la ausencia de consentimiento informado. La Audiencia Provincial aún reconociendo la falta de información al paciente afirma que esta no genera "responsabilidad por el estado del paciente no sólo porque ya había sufrido otra intervención de igual naturaleza, si bien a través de la ingle (vía femoral) que para el perito (...) entraña más riesgo (...), sino también porque además la prueba se realizó,

${ }^{3}$ Cfr. Antecedente 2c. 
como se razona por la Juzgadora en su fundamento de derecho quinto en un momento en el que existía riesgo vital ante la situación que le llevo a urgencias, y que se palió, como ya se ha razonado, con la intervención"4.

El demandante, según recoge el antecedente 3 demanda el amparo sobre la base de la vulneración de su derecho a la tutela judicial efectiva del artículo 24 de la Constitución en relación con los derechos de integridad física del artículo 15 y de libertad del artículo 17.1 de la Constitución, y lo hace dado que las dos sentencias precedentes le niegan la indemnización pese a reconocer que no hubo consentimiento. El recurrente sostiene la infracción de lo establecido en el artículo 8 de la Ley de autonomía del paciente y en el Convenio del Consejo de Europa sobre Derechos del Hombre y la Biomedicina y en la propia Constitución, textos de los que deduce la obligación legal de informar sobre las consecuencias relevantes habituales de todo acto médico, salvo en el caso de riesgo grave e inmediato, supuesto que no concurría en el caso de autos, donde existió un lapso de tiempo suficiente entre el ingreso del paciente en urgencias y la práctica del cateterismo, que se realizó al día siguiente, cómo para que se le diera información sobre el procedimiento a realizar y él pudiera autorizarlo.

Por su parte, la Agrupación Mutual Aseguradora formuló sus alegaciones interesando la desestimación, y recuerda que el paciente fue objeto de un cateterismo arterial en el año 1994, por lo que era perfecto conocedor de la intervención, naturaleza, riesgos etc., y, por otro lado, resulta indudable declarar la urgencia con la que se llevó a cabo la intervención, ya que la noche anterior el paciente sufría importantes complicaciones cardiacas que conllevaron su urgente realización. Insiste también en que los médicos de la Mutua demandad tienen su primer contacto con el paciente en el momento en que se le baja de la UVI para ser intervenido, y que deberían haber sido los médicos de la UVI que le atendieron hasta ese momento los que le hubieran informado de los riesgos de la prueba ${ }^{5}$.

En cuanto a la posición del Fiscal, esta adelanta de alguna manera el que será el pronunciamiento del Tribunal. El Fiscal interesará el otorgamiento de amparo por entender vulnerado el derecho a la integridad física del demandante, centrándose en la carencia de información al paciente, en relación con el artículo 15 de la

\footnotetext{
${ }^{4}$ Cfr. Antecedente 2d.

${ }^{5}$ Cfr. Antecedente 6.
} 
Constitución sobre la dignidad de la persona, que implica "la prohibición de intervenir en el cuerpo de otra persona sin el previo consentimiento de esta, siendo preciso para obtenerlo una previa información suficiente que abarque la expresión del acto invasor y sus posibles consecuencias"6.

Se apoya para mantener este criterio en la Carta de Derechos Fundamentales de la Unión Europea, en el Convenio Europeo de Derechos Humanos y en el tratamiento dado a la cuestión por el Tribunal de Derechos Humanos, así como en el desarrollo legal del consentimiento informado en España, sobre la base de la Ley de autonomía del paciente, así como en la doctrina del Tribunal Supremo y del Tribunal Constitucional en lo relativo al consentimiento informado y al derecho fundamental a la integridad física respectivamente, a saber: SSTC 120/1990, 207/1996, y 154/2002, así como AATC 192/1993 y 57/2007.

Puede decirse a estos efectos que ya está maduro en España a nivel legal, doctrinal y jurisprudencial, el tema del consentimiento informado, después de un desarrollo jurisprudencial de veinte años, en los que la institución ha ido abriéndose paso venciendo las resistencias de algunas sentencias que utilizaban argumentos como la urgencia de la intervención, la necesidad de la actuación sanitaria, la corrección técnica de la intervención, la ficción de la persona razonable que hubiera consentido de haber sido informada etc. , para pasar por alto el consentimiento. Argumentos esgrimidos desde una perspectiva ideológica de resistencia basada en el recelo al libre ejercicio de la autonomía del paciente por una especial consideración de la actuación médica, así como de la vida y de la salud de la persona y del tratamiento jurídico que se le debe dar.

El Fiscal abordará precisamente la forma en que las sentencias recurridas tratan el tema del consentimiento del paciente y la manera en que estas sentencias convalidan la inexistencia de información y consentimiento en el caso de autos. Cómo lo hacen no resulta justificado para el Fiscal, habida cuenta de que "la naturaleza y el rango del derecho fundamental en juego... requiere una fundamentación motivadora de índole reforzada". De modo que a juicio del Fiscal ni la urgencia de la intervención, que según su criterio no fue tal, ni el riesgo vital de la intervención, ni la mera alusión a la edad del paciente, ni el hecho de haber padecido un cateterismo con

\footnotetext{
${ }^{6}$ Cfr. Antecedente 8.
} 
anterioridad, ofrecen descargo suficiente para omitir el deber de informar y recabar el consentimiento. Del mismo modo que no es suficiente la entrega de una hoja formulario a la hora del alta con indicaciones de observación y tratamiento postoperatorio, que no podía cumplir con el deber de obtener previamente a la intervención un consentimiento debidamente informado. El Fiscal concluye que los médicos que indicaron o practicaron la intervención incumplieron sus obligaciones entorno a la información y al consentimiento del paciente, apreciando que las sentencias recurridas omiten toda referencia normativa en sus razonamientos que justifiquen el incumplimiento de este deber de consentimiento, por lo que debe sostenerse que vulneraron el derecho fundamental a la integridad física.

\section{2 ¿Cuál es el posible derecho vulnerado?}

Precisamente, y ya en los fundamentos jurídicos, el Tribunal Constitucional se plantea si en el caso de autos se produjo una violación de los derechos fundamentales del actor como consecuencia de la falta de consentimiento informado previa a la realización de la intervención sanitaria que le produjo la pérdida funcional total de la mano derecha. El actor solicita el amparo basándose en los artículos 15 y 17.1 de la Constitución en relación con el artículo 24 de la Constitución, relativo al derecho a la tutela judicial efectiva, que habría sido vulnerada por la desestimación de su pretensión en las dos instancias anteriores.

El Tribunal Constitucional va a pronunciarse entonces sobre si la actuación sanitaria que está en la base de la reclamación del actor produjo o no la vulneración de los derechos fundamentales invocados por el reclamante ${ }^{7}$.

Como vimos el demandante reclama el amparo sobre la base de los derechos a la integridad física y a la libertad (arts. 15 y $17.1 \mathrm{CE}$ ) pero el Tribunal solo examina el primero ya que considera, siguiendo su reiterada doctrina, que la libertad protegida por el art. 17.1 es la "libertad física", es decir la libertad frente a la detención, condena o internamiento arbitrario ${ }^{8}$. Así se concreta el contenido del artículo 17.1 sin que

\footnotetext{
${ }^{7}$ En este punto el Tribunal aclara que éste es el objeto de su pronunciamiento, y no la procedencia o no de la indemnización reclamada, ya que esta es una cuestión de legalidad ordinaria cuya apreciación es labor exclusiva de los órganos de la jurisdicción ordinaria en el ejercicio de la función que les atribuye el artículo 117.3 de la Constitución.

${ }^{8}$ Según jurisprudencia reiterada del Tribunal, como puede verse en STC 126/1987, 22/1988, 112/1988, y $61 / 1990$.
} 
según el Tribunal Constitucional pueda cobijarse en el mismo una libertad general de actuación o una libertad general de autodeterminación individual. Según establece el Tribunal Constitucional esa clase de libertad es un valor superior del ordenamiento jurídico que aparece recogido en el artículo 1.1 de la Constitución y sólo tiene la protección del recurso de amparo en aquellas concretas manifestaciones a las que la Constitución les concede la categoría de derechos fundamentales incluidos en el capítulo segundo de su título I, como son las libertades a que se refiere el propio artículo 17.1 y los artículos $16.1,18.1,19$ y 20, entre otros ${ }^{9}$.

El Constitucional recuerda que ya en la Sentencia 89/1987 había distinguido entre las manifestaciones "de la multitud de actividades y relaciones vitales que la libertad hace posibles", que denomina también manifestaciones de la "libertad a secas", y "los derechos fundamentales que garantizan la libertad", pero que "no tienen ni pueden tener como contenido concreto cada una de esas manifestaciones en su práctica, por importantes que sean estas en la vida del individuo". Es decir que se produce una selección entre las manifestaciones de la libertad individual de manera que sólo algunas de ellas tendrán la consideración de derechos fundamentales.

Como consecuencia el Tribunal va a situar exclusivamente en el artículo 15, relativo a la integridad física y moral, y no ya en el artículo 17.1, la posible vulneración de un derecho fundamental en el caso de actuación sanitaria llevada a cabo sin el consentimiento del paciente. De esta manera lo que se considera que puede haber sido vulnerado en el caso de autos es la realización de una intervención sin informar previamente al paciente de sus riesgos y posibles consecuencias no es la libertad en abstracto del sujeto, sino una específica manifestación de esa libertad que es en este caso la integridad física y moral de la persona protegida por el artículo 15 de la Constitución ${ }^{10}$.

\section{3 ¿Cuál es el contenido del derecho que se invoca?}

Así pues el análisis del Tribunal va a ceñirse a si nos encontramos ante una lesión del derecho a la integridad física y moral, derecho amparado de forma autónoma y con sustantividad propia en el artículo 15 de la Constitución.

\footnotetext{
${ }^{9}$ Cfr. F. J. $3^{\circ}$.

${ }^{10}$ Cfr. Ibidem.
} 
Se considera entonces que el análisis debe referirse a si la intervención realizada sin informar al paciente previamente de sus riesgos y posibles consecuencias ha supuesto o no una lesión a su derecho a la integridad física y moral del artículo 15 de la Constitución, que adquiere de esta forma según el Tribunal una sustantividad propia.

Se empieza entonces por analizar el contenido del artículo 15, que según la doctrina del propio Tribunal, en sentencias 220/2005 de 12 de septiembre y 160/2007 de 2 de julio, protege "la inviolabilidad de la persona, no sólo contra ataques dirigidos a lesionar su cuerpo o espíritu, sino también contra toda clase de intervención en esos bienes que carezca del consentimiento de su titular (sentencias 120/1990 de 27 de junio y 119/2001 de 24 de mayo). Se dice que estos derechos "destinados a proteger la incolumidad corporal (sentencia 207/1996 de 16 de diciembre) han adquirido también una dimensión positiva en relación con el libre desarrollo de la personalidad, orientada a su plena efectividad, razón por la que se hace imprescindible asegurar su protección no sólo frente a las injerencias ya mencionadas, sino también frente a los riesgos que puedan surgir en una sociedad tecnológicamente avanzada (sentencias 119/2001 de 24 de mayo). De ahí que para poder apreciar la vulneración del artículo 15 de la Constitución no sea preciso que la lesión de la integridad se haya consumado, sino que basta con que exista un riesgo relevante de que la lesión pueda llegar a producirse (sentencia 221/2002 de 25 de noviembre)"11.

Además de ello, continúa diciendo el Tribunal, "hemos afirmado que el derecho a que no se dañe o perjudique la salud personal queda también comprendido en el derecho a la integridad personal (sentencia 35/1996 de 11 de marzo)", aunque aclara también que "no todo supuesto de riesgo o daño para la salud implique una vulneración del derecho fundamental, sino tan sólo aquel que genere un peligro grave y cierto para la misma (sentencias 119/2001 de 24 de mayo y 5/2002 de 14 de enero)" ${ }^{12}$.

Para el Tribunal Constitucional "el derecho fundamental a la integridad física y moral conlleva entonces también una facultad negativa, que implica la imposición de un deber de abstención de actuaciones médicas salvo que se encuentren

\footnotetext{
${ }^{11}$ Cfr. Ibidem.

${ }^{12}$ Cfr. Ibidem.
} 
constitucionalmente justificadas, y, asimismo, una facultad de oposición a la asistencia médica, en ejercicio de un derecho de autodeterminación que tiene por objeto el propio sustrato corporal, como distinto del derecho a la salud o a la vida (sentencia 154/2002 de 18 de julio). Por esa razón, hemos afirmado que el derecho a la integridad física y moral resultará afectado cuando se imponga a una persona asistencia médica en contra de su voluntad, que puede venir determinada por los más variados móviles y no sólo por el de morir y, por consiguiente, esa asistencia médica coactiva constituirá limitación vulneradora del derecho fundamental a la integridad física, a no ser que, como hemos señalado, tenga una justificación constitucional (sentencias 120/1990 de 27 de junio y 137/1990 de 19 de julio)" ${ }^{13}$.

Este párrafo es clave para entender el sentido que otorga el Tribunal Constitucional al derecho a la integridad física y moral, como distinto del derecho a la salud y del derecho a la vida. Relevante distinción entre el derecho a la salud y el derecho a la integridad física, que aparece remitir, insistiendo en una línea jurisprudencial ya consolidada, a la existencia de este último derecho aún en el caso de que su ejercicio pueda considerarse contrario a una consideración objetiva de la salud del sujeto.

Su interpretación supone que lo que se protege es el derecho de la persona a la incolumidad corporal, al respeto a su integridad corporal, a que no se pongan las manos sobre su cuerpo, a qué no se intervenga sobre su cuerpo sin su consentimiento, y por lo tanto a rechazar tratamientos médicos aún siendo estos beneficiosos para la salud, siempre que no sean constitucionalmente obligatorios.

El cuerpo aparece así como una esfera privada libre de intervención, como un santuario que se planta incluso frente a la actuación sanitaria en beneficio de la salud del paciente. De esta manera el Tribunal Constitucional ampara el derecho a la autonomía del paciente por encima del paternalismo médico.

Una vez dejado claro este posicionamiento abordamos la duda respecto del caso de autos, en el que no se da propiamente una asistencia médica coactiva, sino una intervención sanitaria tendente a mejorar el estado de salud del paciente precisamente a instancias del propio paciente, que se encontraba aquejado de complicaciones cardiacas. Lo que ocurre es que en este caso no se dio al paciente la

\footnotetext{
${ }^{13}$ Cfr. Ibidem.
} 
debida información sobre la naturaleza y los riesgos de la intervención, ni se produjo su posterior consentimiento.

Por eso el Tribunal Constitucional se ve en la tesitura en la que queríamos encontrarle, que no es otra que la de considerar si la omisión del consentimiento informado del recurrente en el caso de una intervención médica pensada a favor de su salud, implica o no una lesión de su derecho a la integridad física.

Para ello el Tribunal lo que hará será enfrentarse a la naturaleza de ese consentimiento informado, con la finalidad de concretar si forma parte del derecho fundamental a la integridad física y moral del artículo 15 de la constitución Española, según el contenido de este derecho que se ha expuesto con anterioridad.

\section{La naturaleza del consentimiento informado.}

Vemos cómo el caso de autos lleva pues al Tribunal Constitucional a plantearse la naturaleza del consentimiento informado, y con ello a pronunciarse sobre el sentido constitucional de esta institución, que desde hace veinte años viene experimentando un progresivo desarrollo legal y jurisprudencia.

A la hora de plantearse esta cuestión el Constitucional sostendrá que aunque el artículo 15 no contiene una referencia al consentimiento informado, esto no implica que este instituto quede fuera de de la previsión constitucional de protección de la integridad física y moral. La sentencia se remite a pronunciamientos anteriores (sentencias 212/1996 de 19 de diciembre y 116/1999 de 17 de junio) para reiterarse en la afirmación conforme a la cual "los preceptos constitucionales relativos a los derechos fundamentales y libertades públicas pueden no agotar su contenido en el reconocimiento de los mismos, sino que, más allá de de ello, pueden contener exigencias dirigidas al legislador en su labor de continua configuración del ordenamiento jurídico, ya sea en forma de principios rectores de contornos más amplios, ya sea, como enseguida veremos, en forma de bienes jurídicos constitucionalmente protegidos" ${ }^{14}$. Idea que se refuerza con el recordatorio de que de la obligación de sometimiento de todos los poderes públicos a la Constitución se deduce tanto la obligación negativa del Estado de no lesionar la esfera individual o institucional protegida por los derechos fundamentales, como también la obligación

\footnotetext{
14 F. J. 4ํㅗ.
} 
positiva de contribuir a la eficiencia de tales derechos y de los valores que representan. $Y$ ello obliga al legislador, especialmente en los casos en que un derecho o valor fundamental quedaría vacío de contenido de no establecerse los supuestos para su defensa.

En este momento cabe plantearse qué implicaciones tiene el derecho a la integridad física y moral en relación con las actuaciones sanitarias. La idea es que, evidentemente, las actuaciones médicas llevan implícita una posibilidad de afectar a la integridad personal protegida por el artículo 15 en la medida en que éste tutela la inviolabilidad de la persona contra toda intervención en su cuerpo. A partir de ahí se trata de arbitrar los mecanismos oportunos para garantizar la efectividad de este derecho en relación con la función y finalidades propias de la actividad médica. Esto se lleva a cabo, según el Constitucional, considerando que "la garantía de efectividad del derecho a la integridad personal en el ámbito médico implica que cualquier actuación que afecte a la integridad personal, para resultar acorde con dicho derecho... se ha de encontrar consentida por el sujeto titular del derecho o debe encontrarse constitucionalmente justificada" ${ }^{15}$. Así es definido en principio el alcance del derecho fundamental a la integridad personal en relación con el consentimiento informado a las actuaciones sanitarias.

Y de ahí que según el Tribunal Constitucional el legislador deba establecer los mecanismos necesarios para la prestación del consentimiento por parte del sujeto en los supuestos de actuaciones médicas sobre su cuerpo, así como los casos en los que constitucionalmente puede prescindirse o matizarse ese consentimiento, teniendo en estos casos en cuenta que los derechos fundamentales sólo pueden ceder ante límites establecidos por la propia Constitución al establecer el derecho, o ante los que se deriven de la necesidad de preservar otros derechos constitucionalmente protegidos.

Nótese que el Constitucional se refiere a "este instituto" cuando analiza si el consentimiento informado está dentro o no de la previsión constitucional de protección de la integridad física y moral, con lo cual ya podemos decir que el consentimiento informado es, al menos, un instituto jurídico.

\footnotetext{
15 Ibidem.
} 
Una vez determinado que el consentimiento informado forma parte del derecho fundamental a la integridad física y moral, el Tribunal constitucional va a proceder a determinar las garantías que se incluyen en ese derecho en relación con las posibles actuaciones sanitarias. Y aunque el art. 15 no contiene referencia expresa al consentimiento informado, el Tribunal Constitucional va a proceder a estudiar el instituto para determinar su contenido en relación con el derecho fundamental.

A partir de ahí el Tribunal Constitucional pasa a determinar las garantías que desde la perspectiva del derecho fundamental a la integridad física se imponen a toda intervención médica que afecte la integridad corporal. Para hacerlo acude a los Tratados y acuerdos internacionales ratificados por España, a la doctrina del Tribunal Europeo de Derechos humanos y a la regulación legal existente en nuestro país ${ }^{16}$.

En cuanto a los tratados y acuerdos internacionales ratificados por España, se señala que tienen, de acuerdo con el artículo 10.2 de la Constitución (citando por todas la sentencia del propio Tribunal 6/2004 de 16 de enero), valor interpretativo de las normas relativas a los derechos fundamentales y las libertades públicas, como ocurre con la doctrina del Tribunal Europeo de Derechos Humanos, de acuerdo con el mismo artículo 10.2, según el propio Tribunal recoge en sentencias como la 303/1993 de 25 de octubre y 119/2001 de 24 de mayo.

Entre estos elementos hermenéuticos el Tribunal recurre en primer lugar, en su indagación del contenido del derecho fundamental, a la integridad física y moral a la Carta de los Derechos Fundamentales de la Unión Europea, aprobada en Niza el 7 de diciembre del año 2000 y reconocida con el mismo valor jurídico que los tratados por el artículo 6.1 del Tratado de la Unión Europea (Tratado de Lisboa de 13 de diciembre de 2007, en vigor desde el 1 de diciembre de 2009). La Carta de Niza reconoce en su artículo 3 el derecho de toda persona a la integridad física y psíquica, obligando a respetar, en el marco de la medicina y la biología "el consentimiento libre e informado de la persona de que se trate, de acuerdo con las modalidades establecidas por la ley" 17 .

Acude también al Convenio para la protección de los derechos humanos y la dignidad del ser humano con respecto a las aplicaciones de la Biología y la Medicina, redactado en Oviedo el 4 de abril de 1997 y ratificado por España mediante

\footnotetext{
${ }^{16}$ Cfr. Ibidem.

${ }^{17}$ Cfr. Ibidem.
} 
Instrumento de 23 de julio de 1999, con entrada en vigor el 1 de enero del año 2000. Este Convenio establece en su artículo 5 como regla general que sólo podrá realizarse una intervención en el ámbito de la sanidad "después de que la persona afectada haya dado su libre e informado consentimiento" a cuyo efecto "deberá recibir previamente una información adecuada acerca de su finalidad y la naturaleza de la intervención, así como sobre sus riesgos y consecuencias" ${ }^{18}$.

El Constitucional pasa después a examinar la doctrina del Tribunal Europeo de Derechos Humanos, señalando en primer lugar que el Tratado Europeo de Derechos Humanos no contiene una disposición específica relativa a la protección de la integridad física y moral, pese a lo cual el Tribunal Europeo ha considerado que esta está englobada en la noción de "vida privada" cuyo respeto consagra el artículo 8.1 del Convenio (según sentencias del Tribunal Europeo de 16 de diciembre de 1997, caso Raninen c. Finlandia y de 24 de febrero de 1998, caso Botta c. Italia). El tribunal también ha incluido en el Convenio la participación de los individuos en la elección de los actos médicos de los que sean objeto así como las relativas a su consentimiento, según sentencia de 24 de septiembre de 1992 (caso Herczegfalvy c. Austria y sentencia de 29 de abril de 2002, caso Pretty c. Reino Unido). En suma el Tribunal destaca la importancia del consentimiento de los pacientes y considera que la imposición de un tratamiento médico sin el consentimiento del paciente, cuando este es una adulto sano mentalmente, supone un ataque a la integridad física del interesado que puede poner en cuestión los derechos protegidos por el artículo 8.1 del Convenio.

El Tribunal pone también de relieve la importancia para las personas expuestas a un riego para su salud de tener el debido acceso a la información que les permita evaluar dicho riesgo (Sentencia de 19 de febrero de 1998, caso Guerra u otros c. Italia, y de 2 de junio de 2009, caso Codarcea c. Rumania). El Tribunal llega a sostener que "en su obligación de adoptar normas adecuadas para garantizar el respeto a la integridad física de los pacientes, los Estados parte deben imponer las normas precisas para que los médicos se pregunten sobre las consecuencias previsibles de la intervención médica proyectada sobre la integridad física de sus pacientes e informen a estos convenientemente sobre aquellas, de modo que la

\footnotetext{
${ }^{18}$ Cfr. Ibidem.
} 
persona pueda consentir el acto con conocimiento de causa, de suerte que si se consuma un riesgo previsible sin que el paciente haya sido informado por el médico, el Estado concernido podría llegar a ser directamente responsable al abrigo del artículo 8 del Convenio" 19 .

Como resultado de este análisis de los tratados internacionales y de la doctrina del Tribunal Europeo de Derechos humanos, el Constitucional español va a dejar sentado que "el consentimiento del paciente a cualquier intervención sobre su persona es algo inherente, entre otros, a su derecho fundamental a la integridad física, a la facultad que éste supone de impedir toda intervención no consentida sobre su propio cuerpo, que no puede verse limitada de manera injustificada como consecuencia de una situación de enfermedad" 20.

El Tribunal considera que este derecho se basa en la autodeterminación del paciente, en la autonomía de su voluntad, que le permite decidir libremente sobre las distintas medidas terapéuticas o tratamientos que pueden afectar a su integridad. A partir de ahí el paciente puede escoger entre las distintas posibilidades, consintiendo su práctica o rechazándola.

Para el Constitucional precisamente la manifestación más importante de los derechos fundamentales que pueden resultar afectados por una intervención médica es la de decidir libremente entre consentir un tratamiento o rehusarlo ${ }^{21}$.

Esta última posibilidad se admite aún cuando de la elección pudiera derivarse un resultado fatal, como se recoge en la sentencia del Tribunal Europeo de 29 de abril de 2002, ya citada, o en la del propio Tribunal Constitucional 154/2002 de 18 de julio.

Pero inmediatamente después de dejar sentada la relevancia del consentimiento como manifestación de la autonomía de la voluntad y como contenido del derecho a la integridad de la persona, el Tribunal va a poner de manifiesto como el consentimiento requiere para poder ser ejercido válidamente, de una adecuada información ${ }^{22}$. En efecto la información es objeto también de atención por parte del Tribunal a la hora de abordar el papel del consentimiento. A este respecto se va a señalar que para que se pueda ejercer con plena libertad esa facultad de consentir resulta imprescindible que el paciente cuente con la información médica adecuada

\footnotetext{
${ }^{19}$ Cfr. Ibidem.

${ }^{20}$ Cfr. F. J. 50.

${ }^{21}$ Cfr. Ibidem.

22 Cfr. Ibidem.
} 
sobre las posibilidades terapéuticas. Consentimiento e información son vistos como "dos derechos tan estrechamente imbricados que el ejercicio de uno depende de la previa correcta atención del otro". Como consecuencia la limitación o privación no justificada de la información equivale a una limitación o privación del derecho a consentir, y por lo tanto supone una violación del derecho a la integridad física del que el derecho a consentir es una manifestación.

Para el Constitucional la información previa, que como sostiene ha dado lugar a lo que se ha venido en llamar el consentimiento informado, es un mecanismo de garantía para la efectividad del principio de autonomía de la voluntad del paciente y por tanto de los derechos constitucionales que pueden resultar concernidos por las actuaciones médicas, y señaladamente una consecuencia obligada del derecho a la integridad física y moral. Con ello el derecho a la información alcanza, a juicio del Tribunal Constitucional, una relevancia constitucional que hace que su omisión o defectuosa realización pueda suponer una lesión del propio derecho fundamental.

Esta consideración relativa a la importancia de la información es utilizada por el Tribunal para dar entrada a las referencias legales como criterio para establecer la posición jurídica de la institución del consentimiento informado. Y lo hace señalando como la ley española recoge la importancia de la información precisamente en la Ley 41/2002 de 14 de noviembre, denominada ley básica reguladora de la autonomía del paciente y de los derechos y obligaciones en materia de información y documentación clínica. Una ley que enuncia entre sus principios básicos en su artículo 2 la exigencia, con carácter general, del previo consentimiento de los pacientes o usuarios para toda actuación en el ámbito de la sanidad, "que debe obtenerse después de que el paciente reciba una información adecuada". En la ley queda también recogido el derecho a decidir libremente entre las opciones clínicas disponibles tras recibir la información adecuada, y a negarse al tratamiento, salvo en los casos previstos en la ley. El artículo 4 regula el derecho a la información asistencial de los pacientes, como medio indispensable para ayudarle a tomar decisiones de acuerdo con su propia y libre voluntad. Y hace al "médico responsable" encargado de garantizar esa información, así como a los profesionales que le atiendan durante el proceso asistencial o le apliquen una técnica o un tratamiento concreto. La ley reconoce también el derecho a no recibir información cuando el paciente así lo considere y decide expresamente, sometiéndose entonces al criterio de los facultativos. 
Con este material jurisprudencial y normativo, y habiendo llegado como hemos visto a unas importantes conclusiones en relación con el papel del consentimiento informado como contenido del derecho fundamental a la integridad física del artículo 15, analiza el Tribunal la queja del recurrente en el caso de autos ${ }^{23}$.

El Tribunal tiene por probado, de acuerdo con lo establecido por las propias sentencias de instancia, que no se prestó la información previa sobre la intervención quirúrgica que se iba a practicar, omitiéndose el consentimiento informado. No obstante, esto no quiere decir necesariamente que se vulnerara el derecho a la integridad física del paciente, pues la falta de consentimiento es admisible si se hace justificadamente desde el punto de vista constitucional. Como se sabe ningún derecho fundamental es un derecho absoluto, y podemos encontrarnos con limitaciones a su ejercicio, limitaciones eso sí que deben estar justificadas constitucionalmente.

Ahora bien, el Tribunal constitucional señalará también que tratándose de la integridad física y moral del artículo 15.1 lo que está en juego es un derecho fundamental sustantivo, lo que hace que la tutela judicial sea distinta y más exigente, ya que como dice la jurisprudencia del Tribunal, sobre resoluciones judiciales que inciden en el contenido de un derecho fundamental sustantivo pesa un deber de motivación reforzada, por comparación con el específicamente derivado del derecho a la tutela judicial efectiva proclamado en el art. 24.1. Este plus de motivación está expresamente exigido por la jurisprudencia del Constitucional en sentencias como las 292/2005 de 10 de noviembre y 224/2007 de 22 de octubre.

$Y$ aquí es donde el Tribunal deja sentado que lo que se exige en estos casos es no sólo una resolución motivada y fundada en Derecho, sino que además la resolución sea "coherente con el derecho fundamental que se encuentra en juego" 24 . A este respecto el Tribunal Constitucional aclarará que ese plus de motivación "hace referencia a exigencias de orden cualitativo y no cuantitativo, al ser perfectamente posible que existan resoluciones judiciales que satisfagan las exigencias del meritado artículo 24.1 de la Constitución, pues expresen las razones de hecho y de derecho que fundamenten la medida acordada, pero que, desde la perspectiva del libre

\footnotetext{
${ }^{23}$ Ya a partir del F. J. 6ㅇ․

${ }^{24}$ Cfr. Ibidem.
} 
ejercicio de los derechos fundamentales, no expresen de modo constitucionalmente adecuado las razones justificativas de las decisiones adoptadas..."25.

Vale la pena detenerse un momento en este punto para poner de manifiesto cómo en este párrafo el Tribunal considera que puede haber una decisión motivada y fundada en derecho pero que no sea suficiente por no recoger una coherencia suficiente con el derecho fundamental en juego. Esta coherencia se hace derivar de exigencias cualitativas, no simplemente cuantitativas, que justifiquen de modo adecuado la decisión adoptada. El Tribunal parece establecer así un salto en la argumentación tendente a proteger los derechos fundamentales, distinguiendo entre "exigencias de orden cuantitativo y cualitativo". La argumentación, procedimiento de decisión jurídica que el Tribunal reconoce entonces como propio, se vale de distintos tipos de razones, y hay razones, que tienen que ver con la naturaleza y exigencias de los derechos fundamentales, que han de tener más peso que otras razones jurídicas no relacionadas tan estrechamente con los derechos fundamentales.

$Y$ esto es lo que va a suceder en el caso de autos, en el que el Tribunal reconoce que se han argumentado razones para excluir la necesidad de la información y el consentimiento, pero sostiene que todas estas razones no son suficientes para limitar un contenido esencial del derecho a la integridad física como es la necesidad del consentimiento informado como paso previo a cualquier intervención sobre el cuerpo del paciente ${ }^{26}$.

El Tribunal insiste en la relación entre consentimiento informado y derecho a la integridad física y moral. Insiste en que "la privación de información equivale a una privación o limitación del derecho a consentir o rechazar una actuación médica determinada, inherente a todo derecho fundamental a la integridad física y moral” ${ }^{27}$, y recuerda las exigencias de la Ley de autonomía del paciente y las del Convenio de Oviedo relativo a los Derechos Humanos y la Biomedicina. Recoge también los supuestos en los que la legislación prevé la posibilidad de prescindir de la información, supuestos que han de estar expresamente previstos por la ley, tratándose de excepciones que no son ni indeterminadas ni de consideración extensiva, permitiendo la Ley de Autonomía del paciente la limitación del derecho

\footnotetext{
${ }^{25}$ Cfr. Ibidem.

${ }^{26}$ A partir del F. J. 7ㅇ․

${ }^{27}$ Cfr. Ibidem.
} 
únicamente en casos de carencia de capacidad del paciente para entender la información, o por la existencia acreditada de un estado de necesidad terapéutica ("cuando por razones objetivas el conocimiento de su propia situación pueda perjudicar su salud de manera grave"). Supuestos estos en los que es preciso comunicar a los familiares o personas vinculadas al paciente las circunstancias del caso, y facilitarles a ellos la oportuna información.

$Y$ en cuanto al consentimiento los supuestos en los que se puede exceptuar son también excepcionales según el Tribunal, tal y como lo plasma el legislador, que permite prescindir del mismo para llevar a cabo las intervenciones clínicas indispensables a favor de la salud del paciente tan sólo en los casos de riesgo para la salud pública, y "cuando existe riesgo inmediato y grave para la integridad física o psíquica del enfermo y no es posible conseguir su autorización"28. Y aún en este último supuesto, si las circunstancias lo permiten, se debe consultar a los familiares o personas vinculadas de hecho al paciente.

Como consecuencia el Tribunal insiste en considerar la necesaria autorización mediante el previo consentimiento del paciente, precedido de la correspondiente información, como contenido del derecho fundamental a la integridad física y moral.

A partir de ahí el Tribunal analizará los motivos expuestos por las sentencias recurridas para considerar que en el caso de autos no era necesario el consentimiento informado, rebatiéndolos uno a uno.

Se refiere en primer lugar a la existencia de un cateterismo anterior, realizado en 1994, y con acceso además por otra vía, que no se considera exima del deber de informar en la concreta situación en que se encontraba el paciente en el caso de autos. Considera el Tribunal que este argumento no resulta acorde con el contenido propio del derecho fundamental afectado, ni con la exigencia de una interpretación de la legalidad en el sentido más favorable a la existencia del derecho fundamental. El Tribunal acaba incluso por considerar este argumento como irrazonable.

El argumento de que existiera urgencia en la intervención y que fuera tal que no permitiera recabar el consentimiento, es también rechazado por el Tribunal Constitucional. Se considera que no se aprecia en las sentencias de instancia razonamiento alguno que justifique la imposibilidad de obtener el consentimiento

${ }^{28}$ Cfr. Ibidem. 
informado del paciente, o de consultar a sus familiares o personas vinculadas de hecho con él. Imposibilidad que además se compadece mal con el lapso de tiempo transcurrido desde el ingreso a la intervención. El paciente ingresa a las 14:16 del 4 de septiembre de 2005 y el cateterismo no se practica hasta el día siguiente, de manera que había tiempo suficiente para que se hubiera informado al paciente de las características y riesgos de la intervención. Esta circunstancia hace que quede en evidencia la fala de razonamiento en cuanto a los motivos por los que no se facilitó la información ni se recabó el consentimiento.

Tampoco considera el Tribunal que en el caso de autos existiera un riesgo inmediato y grave que justificara el actuar sin informar y recabar el consentimiento del paciente. Un simple riesgo no es suficiente para omitir el deber de recabar el consentimiento. El riesgo ha de estar cualificado por las notas de inmediatez y gravedad, notas que no han sido objeto de mención por las instancias, ni mucho menos han sido objeto de análisis por parte de los órganos jurisdiccionales, que han empleado otros conceptos para justificar que se eludiera la obligatoriedad de la prestación del consentimiento informado (como el de "urgencia relativa"), sin ofrecer "una justificación razonable y ponderada". El Tribunal insiste en que el tiempo transcurrido entre el ingreso y la intervención permitía perfectamente dar cumplimiento a las exigencias legales impuestas en garantía del derecho fundamental a la integridad física del paciente.

En suma el Tribunal Constitucional considera que no se satisfizo el derecho del paciente a prestar el consentimiento debidamente informado y por tanto que se vulneró su derecho fundamental a la integridad física. Las normas concernidas han sido interpretadas y aplicadas por las sentencias recurridas sin tomar en consideración la vinculación entre el deber de informar y recabar el consentimiento con un derecho fundamental, y por lo tanto de manera contraria a la mayor efectividad del derecho. Por ello considera el Tribunal que procede otorgar el amparo.

\section{Consecuencias jurídicas del pronunciamiento constitucional.}

Como resultado de este pronunciamiento del Tribunal Constitucional relativo a la consideración del consentimiento informado como un derecho fundamental y en relación con la jurisprudencia hasta el momento existente en materia de consentimiento informado habría que señalar algunas consecuencias. 
En primer lugar la afirmación en el fundamento jurídico tercero de que para poder apreciar la vulneración del artículo 15 de la constitución no es preciso que la lesión a la integridad se haya consumado, sino que basta con que exista un riesgo relevante de que la lesión pueda producirse.

En segundo lugar que para que implique una vulneración del derecho fundamental el riesgo o daño para la salud ha de ser tal que genere un peligro grave y cierto.

En tercer lugar que los derechos fundamentales sólo pueden ceder ante límites establecidos en la propia Constitución o ante los que se deriven de la necesidad de preservar otros derechos constitucionalmente protegidos.

Cuarto, que de acuerdo con la jurisprudencia del Tribunal Europeo de derechos humanos y siendo obligación de los Estados miembros adoptar las normas para que la integridad física de los pacientes sea respetada, los Estados podrían llegar a ser responsables al abrigo del artículo 8 del Convenio.

$Y$ finalmente que sobre decisiones judiciales que inciden sobre un derecho fundamental pesa un deber de motivación reforzada, debiendo la resolución ser coherente con el derecho fundamental que se encuentra en juego. Existe pues la exigencia de un plus de motivación que hace referencia exigencias de orden cualitativo y no cuantitativo. 